\title{
Research on Children's Education App Network Transmission Based on 5G Mobile Computing Technology
}

\author{
Mou Tangjuan $\mathbb{D}^{1,2}$ and Kim Il-Tae ${ }^{1}$ \\ ${ }^{1}$ Art and Sports, Chosun University, Gwangju Metropolitan 61448, Republic of Korea \\ ${ }^{2}$ School of Digital Arts and Media, Shandong University of Art and Desing, Jinan, Shandong 250001, China \\ Correspondence should be addressed to Mou Tangjuan; mtj@sdada.edu.cn
}

Received 30 December 2021; Accepted 18 January 2022; Published 8 February 2022

Academic Editor: Man Fai Leung

Copyright (c) 2022 Mou Tangjuan and Kim Il-Tae. This is an open access article distributed under the Creative Commons Attribution License, which permits unrestricted use, distribution, and reproduction in any medium, provided the original work is properly cited.

\begin{abstract}
With the continuous improvement of China's scientific and technological level, the popularity of mobile network technology is becoming more and more widespread. Many big data technologies and wireless sensor technologies have brought new changes to people's lives. At present, the functions of communication technology and network transmission have moved from perfection to new innovation and development. 5G mobile network technology has played an important role in various fields. When China's economy and science and technology develop better and better, people pay more and more attention to children's education. The research on children's education has become a hot topic. From the above background, this paper studies the network transmission function and network transmission path of children's education app in $5 \mathrm{G}$ era mobile computing technology. First, starting from the development trend and main functions of 5G, this paper briefly describes the functional requirements of children's education app. The efficient data transmission in the $5 \mathrm{G}$ mobile wireless sensor network is applied to children's education app. Starting from the distance of network transmission nodes, the data transmission optimization algorithm is adopted, and the network transmission module is composed of the best transmission path of nodes. Combined with the energy-saving effect of mobile computing technology, the problem of excessive consumption of network transmission resources is optimized. Finally, the data transmission path is studied in the 5G mobile network. The results show that 5G mobile computing technology can optimize the network transmission performance, improve the operation efficiency of the server, and has a good effect in solving the problems of delay and low coverage. In the process of children's education app network transmission under 5G mobile computing technology, it can ensure the user's personal information and improve the security of system feedback.
\end{abstract}

\section{Introduction}

The existing 4G network has been widely used in the Internet of things and is constantly developing to meet the application needs of the Internet of things in the future. New applications and business models of the future Internet of things need new performance standards, such as large-scale connection, security, reliability, wireless communication coverage, ultralow delay, high throughput, etc. In order to meet these needs, $5 \mathrm{~g}$ technology is expected to provide new interfaces for future Internet of things applications. $5 \mathrm{~g}$ will not only provide 1000 times of Internet traffic growth in the next decade but also provide various industries with basic technologies to support the development of the Internet of things. (1) The Internet of things can promote classroom teaching, realize the interaction between students and students and students and machines through sensor technology, provide an intelligent environment for teaching, improve the traditional learning environment and learning methods, and adjust the teaching structure at any time. (2) The Internet of things can be used for extracurricular learning. It supports extracurricular research, mobile learning, and ubiquitous learning to enrich extracurricular learning methods and promote the development of students' ability. (3) The Internet of things can also be used for school management such as attendance management, school 
library management, teaching instruments, and equipment management and school education safety management, so as to innovate school management means and improve management efficiency and quality.

With the rising level of science and technology in China, the coverage effect and popularity of mobile network are gradually expanding. People's daily life has been inseparable from the support of Internet and mobile technology [1]. Although the traditional $4 \mathrm{G}$ network can meet our daily use, it has obvious defects in the use of intelligent devices and intelligent terminals $[2,3]$. Therefore, the advent of $5 \mathrm{G}$ mobile network has brought new opportunities and changes to network transmission in all walks of life [4]. At present, this technology is in the preliminary development stage and has a good application prospect. In practical applications, many communication industries and electronic information industries have predicted it. With the research upsurge of 5G technology in various countries, many scholars found that compared with $4 \mathrm{G}$ network technology, this technology has obvious efficiency in the use of intelligent devices [5]. In the process of using $5 \mathrm{G}$ mobile network and related technologies, it is necessary to make a reasonable selection of network environment and equipment [6]. We need to integrate more advanced technology and imported equipment to build it. With the advent of $5 \mathrm{G}$ era, our country has also significantly improved in the process of base station construction, and the number and scale are showing a positive upward trend [7]. In the network transmission, we also need to consider the urban area coverage effect and establish a large-scale network structure for the whole area. Therefore, the regional network using 5G mobile network and communication technology can be faster and more stable and has good performance in coverage [8].

At present, children's education app has become the main application in the field of education in China [9]. With the rapid development of mobile network technology, all walks of life have formed their own professional app applications [10]. With the rapid improvement of people's living standards, people pay more and more attention to children's education industry. Children's education, with children as the main body and education as the main purpose, has become the focus of every family [11, 12]. When children's education combines intelligent devices and mobile networks to form the current form of app, it has been widely used in the field of education [13]. This educational app can use mobile phones or computers as the carrier of interactive devices to realize the interactive process between children and teachers. Use the network equipment as the medium to connect the data transmission function [14]. Therefore, children's education app has high research value and important significance. As educational software has become the focus of research, the network transmission performance and process in actual operation have also become the main research content [15].

Starting from the development trend and main functions of $5 \mathrm{~g}$, this paper briefly describes the functional requirements of children's education app. Research and innovation contributions include the following: (1) using the data transmission optimization algorithm, the network transmission module is composed of the best transmission path of the node. Combined with the energy-saving effect of mobile computing technology, the problem of excessive consumption of network transmission resources is optimized. (2) The data transmission path in the $5 \mathrm{~g}$ mobile network is studied. The results show that $5 \mathrm{~g}$ mobile computing technology can optimize the transmission performance of the network, improve the operation efficiency of the server, and have a good effect in solving the problems of delay and low coverage. (3) In the process of children's education app network transmission under $5 \mathrm{~g}$ mobile computing technology, it can ensure the user's personal information and improve the system feedback security.

This paper is mainly divided into three parts. The first part analyzes the development of $5 \mathrm{G}$ mobile computing network and the research status of children's education app in various countries. The second part first combines wireless communication technology with $5 \mathrm{G}$ mobile computing to analyze the transmission process of educational app network. Aiming at the optimization and improvement of data processing in network transmission, the tree structure is used to simulate the transmission algorithm in big data environment. The optimal transmission process is calculated according to the distance between network transmission nodes. The energy-saving link of $5 \mathrm{G}$ network and mobile computing in network transmission is studied. Finally, the factors affecting the efficiency of network transmission are analyzed, and the optimal transmission path of children's education app network under $5 \mathrm{G}$ mobile computing technology is explored. The third part first analyzes the results of the research on the network transmission of children's education app under the mobile network technology and the network transmission path.

\section{The Related Works}

In the construction of $5 \mathrm{G}$ mobile computing network, a variety of schemes and requirements need to be met. The construction of network transmission needs to include whether there is a source, and the cost requirements of the two options for network transmission are also different [16]. In the overall application process, it is necessary to consider whether the efficiency of information resource transmission will increase the actual cost after accessing the 5G network. In the application of reducing network transmission cost, it is also necessary to ensure network security performance [17]. In order to improve the network construction and transmission quality, we need to start from the broadband configuration and network structure and choose a flexible design that can ensure the needs of educational app [18]. Research and development shall be carried out in strict accordance with the network transmission protocol and the functional requirements of children's education app. Due to the complexity of the network structure, different requirements and performance have very different requirements for the level. In the specific implementation process, it is necessary to not only pay attention to the existing network structure but also use wireless communication technologies such as big data to support the $5 \mathrm{G}$ network environment 
[19]. At present, China's network transmission structure has many forms. With the continuous development of mobile technology, in order to promote transmission efficiency and applicability, we need to consider it in 5G environment [20]. Select the environment with strong coverage in the application scenario, maximize the popularity of children's education app network by using $5 \mathrm{G}$ transmission performance, and ensure that the network application can realize the function of cross regional and cross domain transmission. Bring better experience and services for users and children [21].

The development of mobile technology and Internet in the United States is relatively advanced, and people are familiar with the integration of Internet into daily life and work [22]. The idea of mobile learning is also widely recognized in American universities. This kind of learning behavior has had a good effect in children's education schools. They use mobile applications to build a children's learning platform and system, so that mobile learning has achieved good results in the field of children's education [23]. It can not only help children learn knowledge better, but also be free from the constraints of time and place, and realize the real-time interaction with teachers.

The development of online education resources and mobile network devices in Japan is also relatively rapid [24]. With the popularization of intelligent equipment, online library and other applications have enriched people's daily life. Many colleges and universities have realized online teaching and after-school learning services. Educational app software improves students' learning efficiency in their daily life and shows information-based and intelligent learning resources to students in need. In the field of children's education, they first chose platforms such as online counseling as the basis. Subsequently, with the rapid development of mobile technology, online counseling was gradually transformed into the form of mobile application software, which brought a lot of help to children's learning and cognitive society [25].

The scale of Internet users in China has become the top form in the world, including hundreds of millions of mobile phone users. Using mobile phone to surf the Internet has become a common living habit. With the advent of the $5 \mathrm{G}$ network era, it is more convenient for everyone to obtain information by using mobile phones and mobile terminals. In the field of education, network counseling, distance teaching, and other modes have become widely used. People also put forward the idea of intelligent app in the development of children's education software. Many educational institutions have specially developed educational apps to help students expand their knowledge structure according to children's habits and learning frequency. Many children's education apps can also be combined with virtual devices to form a 3D live learning atmosphere. Based on the development status of children's education app in various countries, this paper studies the network transmission performance and path of education app by using mobile computing technology and wireless network technology in $5 \mathrm{G}$ environment.

\section{Research on Children's Education App Network Transmission and Optimal Path Based on 5G Mobile Computing Technology}

3.1. Research on Transmission Optimization Algorithm of Children's Education App Network Based on 5G Mobile Computing Technology and Wireless Sensor Network. To research on network transmission optimization algorithm of children's education app based on $5 \mathrm{G}$ mobile computing technology and wireless sensor network in order to realize the effective application of $5 \mathrm{G}$ mobile network in children's education app, we need to analyze from the actual network application environment. On the basis of coverage, ensure users' excellent sense of experience and efficient information services to meet the needs of most people and children. In order to better realize the network operation, we need to choose a high-capacity environment to provide technical support for traffic transmission and information transmission. In the direction of improving the use effect and popularity of children's education app, it is necessary to choose the operation mode that consumes less resources. Select the technology with less data content and low consumption, and combine it with the education app network transportation module. We study the $5 \mathrm{G}$ network coverage in various regions of the country, as shown in Figure 1.

As can be seen from Figure 1, the national network coverage basically reaches above the standard range. Among them, $5 \mathrm{G}$ in the eastern region develops rapidly and has obvious advantages in coverage. When the $5 \mathrm{G}$ network is selected as the technical support of application software, it is necessary to scientifically analyze the system function. First of all, the user experience should meet the provision of children's learning resources. And resource allocation is carried out for students of different ages. From the interactive experience, we should set some options convenient for children to meet the personalized needs of students. Distinguish the children's operation process from their parents, and set the corresponding parent mode and learning mode. In data management, we should focus on data management and personal information management. Finally, the background mode needs to be established to facilitate the administrator to modify and improve the platform. The specific functional framework is shown in Figure 2.

After analyzing the system functional requirements, we also need to analyze the application status of children's education apps, starting with the acceptance of parents and students. Using the questionnaire and evaluation form, analyze the evaluation coefficients of parents and children on the use experience of children's education app software, as shown in Figure 3.

As can be seen from Figure 3, with the increase of the number of people, we can clearly see that parents generally have a high degree of recognition of children's education app, and students are also satisfied with the actual application process. In the process of network transmission, the transmission of software application generally adopts the digital system. Although this method is relatively stable, it can not guarantee the quality of information and data 


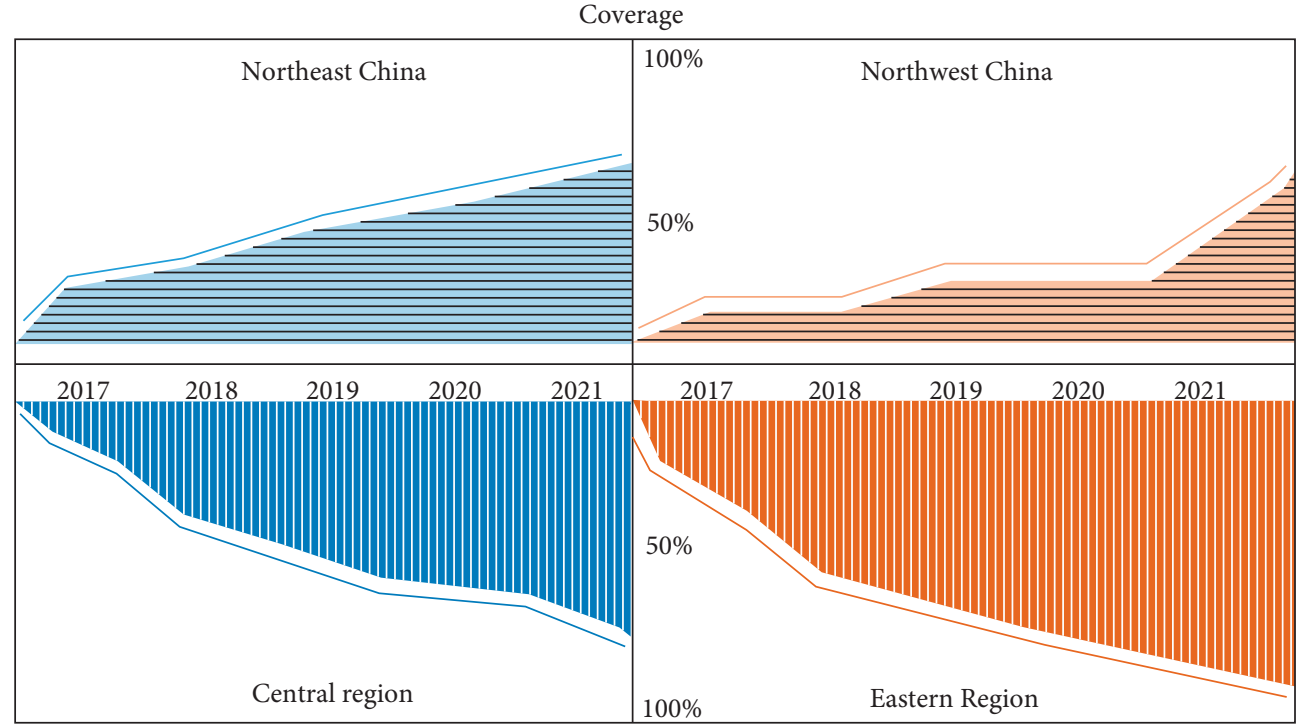

FIGURE 1: 5G network coverage in all regions of the country.

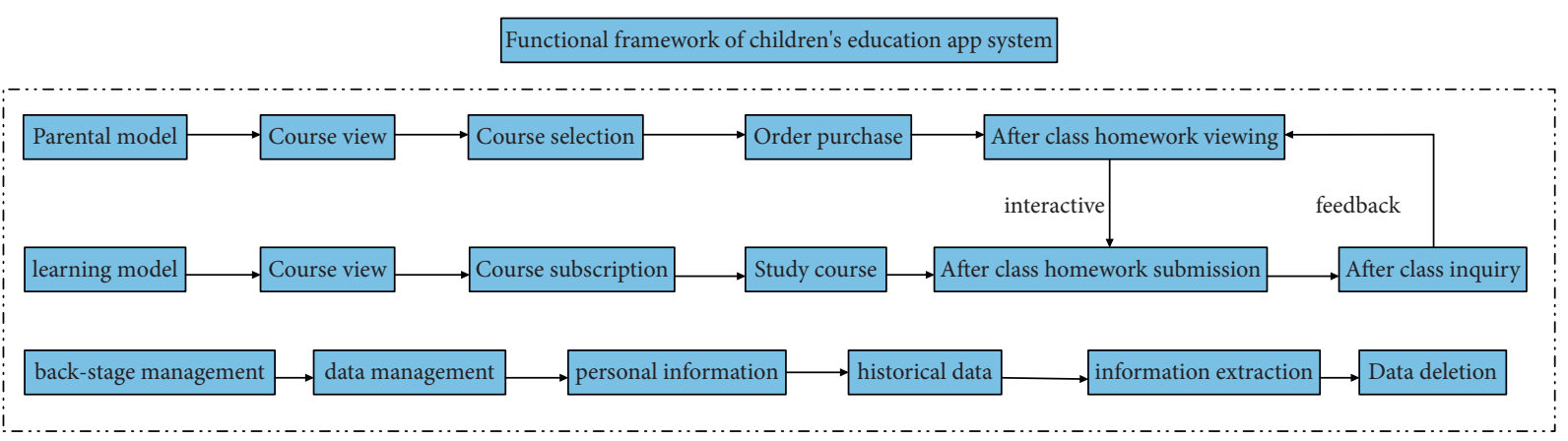

FIgURe 2: Specific functional framework.

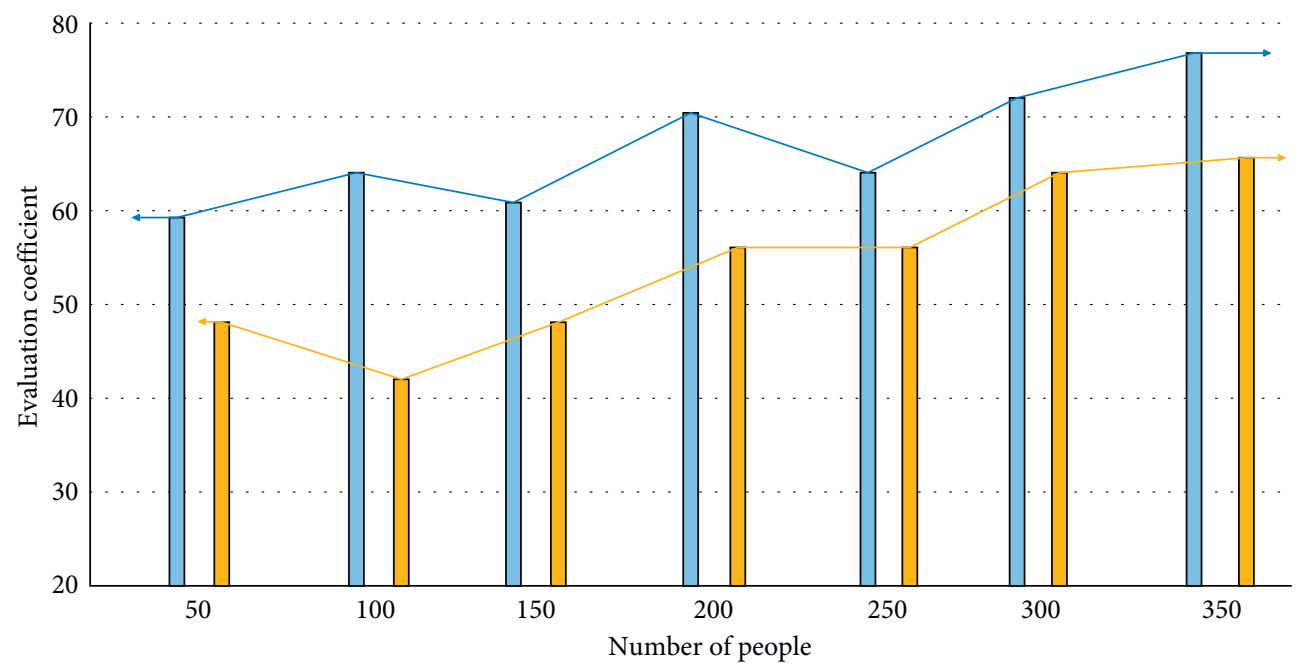

— Parent evaluation coefficient

Child evaluation coefficient

FigURE 3: Comparison of APP use experience between parents and children. 
transmission in the big data environment. With the rapid development of wireless sensor technology and mobile network, many mobile computing technologies in 5G environment have also been widely used. Based on the mobile computing network, this paper analyzes the transmission process and the factors affecting the performance. First, different data nodes are used for distribution in the network model. It is assumed that the data flow consumed by each node is as follows:

$$
\begin{aligned}
& E_{t r}=l * E_{\text {elec }}+l * \varepsilon_{f s} * d^{2}, \quad d \leq d_{0}, \\
& E_{t r}=l * E_{\text {elec }}+C * \varepsilon_{m p} * d^{3}, \quad d>d_{0} .
\end{aligned}
$$

The specific formula after calculation is simplified as follows:

$$
d_{0}=\sqrt{\frac{\varepsilon_{f s}}{\varepsilon_{m p}}} .
$$

The energy consumption of a network node receiving a signal is as follows:

$$
E_{r x}=l * E_{\text {elec }} .
$$

The variable $\varepsilon_{f s}$ represents the maximum distance between nodes, and $\varepsilon_{f s}$ represents the specific energy consumed in the transmission process of each data. According to the mobile computing algorithm, we select the network node in a certain area as the test location in advance. Under comprehensive conditions, select places with too much data storage, and calculate the data change value according to the above factors. The energy consumption of each node is represented by the data set:

$$
C H_{\text {candldata }-l}=a *\left(\frac{E_{\text {Remain-1 }}}{E_{\text {Initlal }}}\right)+\beta *\left(\frac{\operatorname{Deg}_{\text {max }}}{\operatorname{Deg}_{1}}\right) .
$$

The parameters $a$ and $\beta$ represent data greater than 0 and less than 0 , respectively. Set the optimal data transmission route to the number and specific location of nodes to form the communication radius:

$$
\begin{aligned}
T_{(n)} & =\frac{p}{1-p *(r * \bmod )} n \in G, \\
\operatorname{rand}\left(v_{i}\right) & <T\left(v_{i}\right) .
\end{aligned}
$$

After the data transmitted through the network is classified, the optimal transmission path is established according to the maximum coverage level and minimum consumption of the link. In order to reduce the communication complexity of mobile computing technology in educational app, we also need to implement multipath concurrency in network transmission. Finally, the contact terminal is improved and $5 \mathrm{G}$ network transmission technology is implemented in the application of children's education app. We draw and analyze the real-time network transmission structure of 5G mobile computing technology, as shown in Figure 4.

As can be seen from Figure 4, in addition to connecting the mobile terminal, the coverage of the core network and the base station also need to be considered and finally ensure the effective operation of each link in the network transmission.

\subsection{Research on Path Optimization of Children's Education} App Network Based on 5G Mobile Computing Technology. With the coming popularity of $5 \mathrm{G}$ in China, it can drive most of the global networks to realize the connection and transmission function. Intelligent devices and intelligent terminals can appear in many forms. 5G mobile computing network programs are becoming more and more advanced and complex. Although the $5 \mathrm{G}$ network system can quickly handle server identification and information transmission, it can not handle the path node optimization in network transmission to a better state. We optimize the design and research of children's education app network path. Select the distributed network server in network path design. This server can connect the virtual environment with the mobile base station to realize the rapid processing and transmission of data. The network coverage is also stronger than the original structure, and the strength of receiving data is above the standard range. In order to meet the real-time interaction performance of APP users, we need to adopt this network structure with high coverage as the choice of the optimal path. Compare the high coverage mobile network with the ordinary mobile network in real-time interactive response, as shown in Figure 5.

As can be seen from Figure 5, the greater the response coefficient, the higher the efficiency of the network in the interaction process. $5 \mathrm{G}$ networks with high coverage are obviously more interactive than ordinary mobile networks. Subsequently, in order to ensure the stability of data information in network transmission, it is necessary to establish relevant models for transmission nodes. During the request process of each data unit, an independent set shall be configured to realize effective division. In the stable transmission demand, the independent data of the transmission request are controlled within the following range, and the expression is as follows:

$$
M_{i}(c)=\frac{i \times q}{(c+1) * G_{i}(c)} .
$$

In the formula, the variable $q$ represents the number of budget steps in the transmission process. Effective selection by allocation method in network data set is as follows:

$$
w_{y}=\sum^{n} M_{i}(c) \times q .
$$

Assuming that the data node in the formula does not schedule the minimum distance, the equilibrium expression of the transmission coefficient is as follows:

$$
\text { Cain }=\sum_{i=1}^{N}\left[g\left(\Delta w_{i}\right)-l\left(\Delta w_{i}\right)\right] \times w .
$$

$l\left(\Delta w_{i}\right)$ represents the priority of the assigned path and can represent the stability change of the network transmission data set. The task is scheduled for each data set 


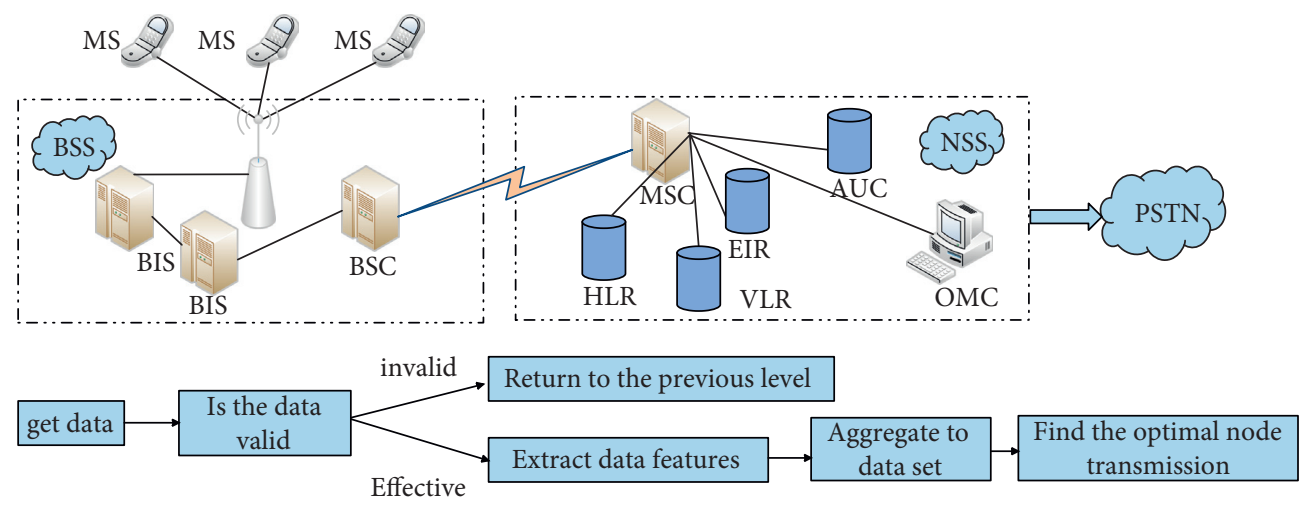

Figure 4: Network transmission structure diagram.

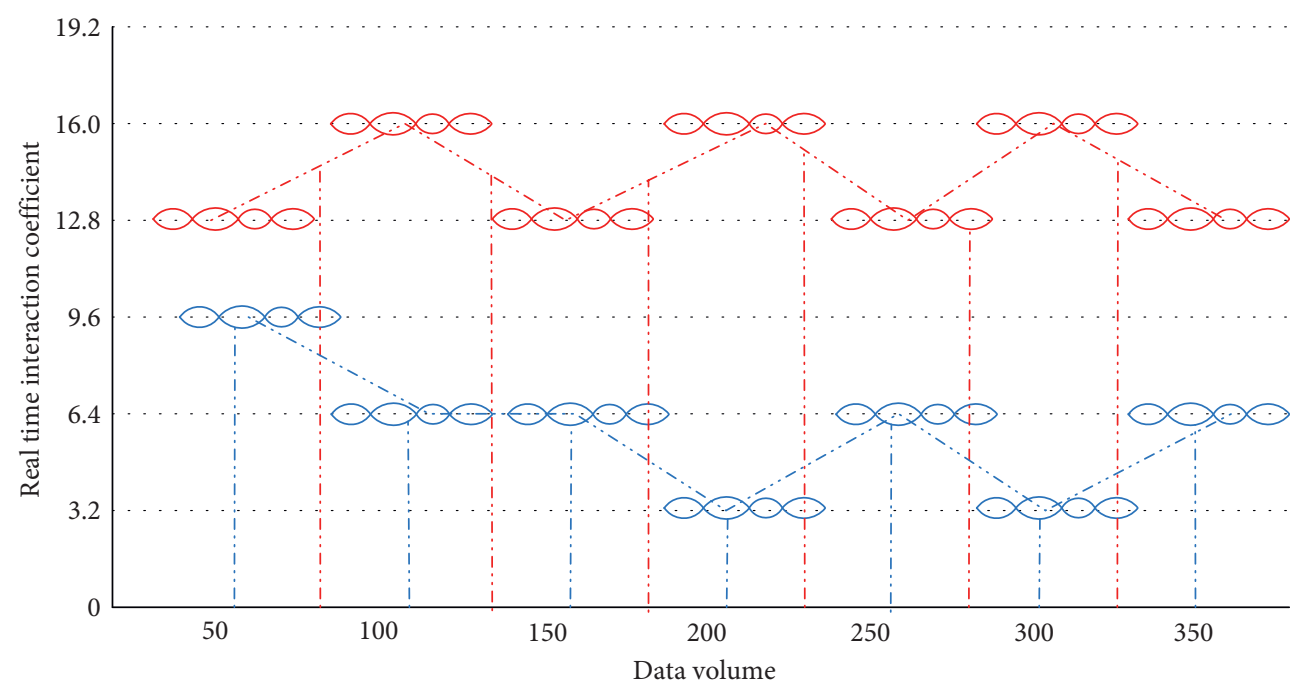

$\infty \infty^{\infty}$ High coverage mobile network $\infty$ General mobile network

Figure 5: Comparison of real-time interactive response between high coverage mobile network and ordinary mobile network.

through sorting calculation, and the minimum coefficient of time is selected as the allocation point. The specific operation model expression is as follows:

$$
C=\frac{\left(D, W^{n}, Q, Z\right)}{(V, W, E, C)}
$$

Among them, the relationship described by the set represented by the data is the change of stability coefficient. It is assumed that the data operation in the model is related to the storage performance:

$$
\begin{aligned}
L_{p} & =G \cdot\left\{\begin{array}{c}
l_{p-1} * p \\
1
\end{array}\right\}, \\
p_{i} & =\bar{T}_{i} \cdot l_{p},
\end{aligned}
$$

where $p_{i}$ is the data size coefficient and $\bar{T}$ represents the number of network transmission nodes. The path of the transmission task is represented according to the order of the above values:

$$
W_{i}=\sum_{n i}^{n i} \frac{w_{i, j}}{p_{i}}
$$

According to the above expression, the processing expression under different states of path in network transmission can be clearly obtained:

$$
\operatorname{rank}\left(n_{i}\right)=\frac{1}{|k|} * \sum_{j=1}^{k} w_{i}\left(n_{i}, p_{j}\right)
$$

In the path optimization of mobile network nodes, in addition to considering the location and communication range of data nodes, it is also necessary to set nodes as marker points. When the access location in data transmission is outside the coverage, we do not need to consider the location of this node, which is called edge node. Compare the position changes of data nodes before and after network transmission path optimization, as shown in Figure 6 . 


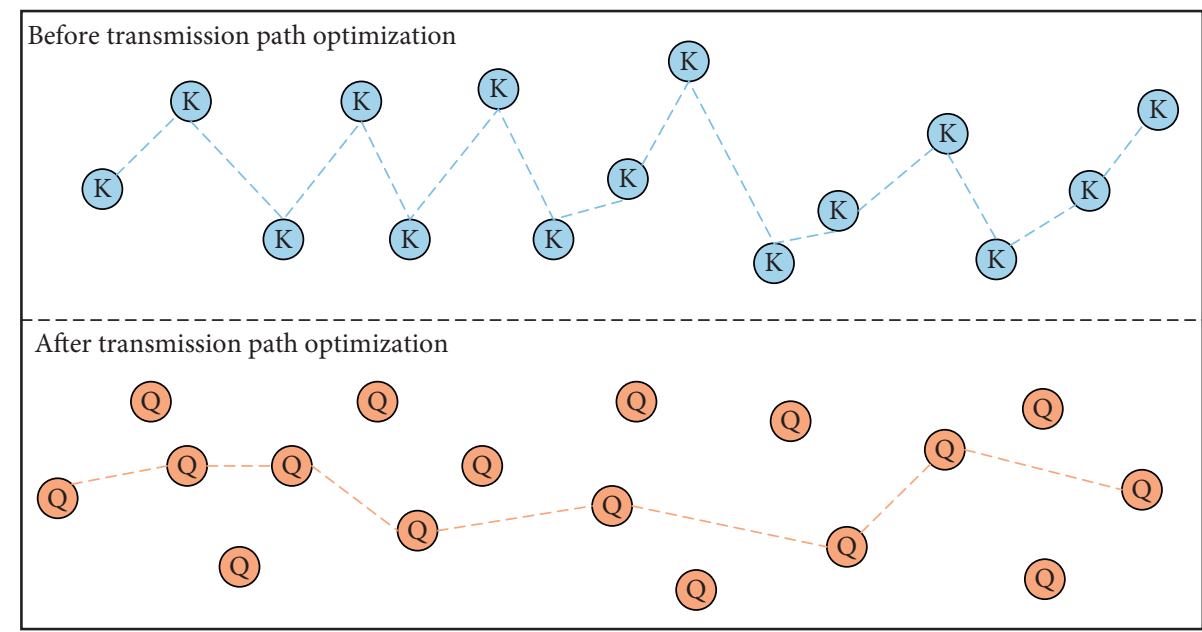

FIgURE 6: Changes of data node position before and after network transmission path optimization.

As can be seen from Figure 6, the node position change after the network transmission path optimization can find the optimal line from multiple location nodes to realize the shortest distance task transmission. Therefore, selecting the optimal network transmission path can effectively improve the operation of the whole platform. Finally, we also need to analyze the transportation consumption of $5 \mathrm{G}$ mobile network. In the process of data flow and information transmission in the service center, there will be different degrees of consumption. According to the system platform load and evaluation data, it can be seen that data transmission consumes the most resources. We should analyze the way in which children's education app consumes the least energy. In addition to the necessary core program operation and peripheral equipment operation, it is also necessary to calculate the access nodes and received data. The energy consumption distribution of children's education app network is shown in Figure 7.

As can be seen from Figure 7, the network consumption is divided into two parts, one is the normal energy consumption in the process of data exchange and the energy consumption in information transmission. The other includes the energy consumption of core programs and terminal devices. In the process of using the children's education app, the long-time standby service will not completely cut off the energy consumption but reduce the network energy consumption and reduce the average consumption. We also need to take the transmission consumption into account in designing the optimal network transmission path.

\section{Analysis of Research Results of Children's Education App Network Transmission and Optimal Path Based on 5G Mobile Computing Technology}

4.1. Analysis of Research Results of Children's Education App Network Transmission Optimization Algorithm Based on 5G Mobile Computing Technology and Wireless Sensor Network.
As a device to realize dynamic interaction and information transmission in software applications, children's education app needs to meet the performance of fast data transmission, smooth data interaction, safe storage location, and so on. With the rapid development of 5G technology, the network transmission process is facing new update and optimization. The transmission process needs to send information and data to users within the network coverage through mobile network lines. In order to meet the people's demand for software signals, the transmission lines and external equipment are also different. When information is sent from the interactive platform, there is an underlying physical channel formed by data flow between the receiver and the sending node. This form can judge the speed that affects the network data transmission. The subsequent wireless network transmission changes this physical channel mode and takes speed and clarity as the core content in information transmission. The transmission speed of children's education app network optimized by mobile computing technology under the $5 \mathrm{G}$ network is compared with that of traditional mobile network, as shown in Figure 8.

As can be seen from Figure 8, APP software using 5G mobile network computing technology has obvious advantages in transmission speed within the same data flow range. The transmission efficiency of traditional mobile networks is not high in the big data environment.

As an important direction of the evolution and upgrading of the new generation of information and communication technology, $5 \mathrm{~g}$ is the key information infrastructure to realize the interconnection of all things and an important driving force for the digital transformation of economy and society. However, while $5 \mathrm{~g}$ technology benefits society and people, it also raises new network security risks. Because mobile providers must support GPRS Tunneling Protocol on their $5 \mathrm{~g}$ network for traditional reasons, users will still be vulnerable to attacks even if the $5 \mathrm{~g}$ protocol itself contains security functions to prevent similar attacks. If it includes user information disclosure (including location data for user tracking), spoofing can be used for fraud and simulated attacks. Denial of service (DOS) attacks on 


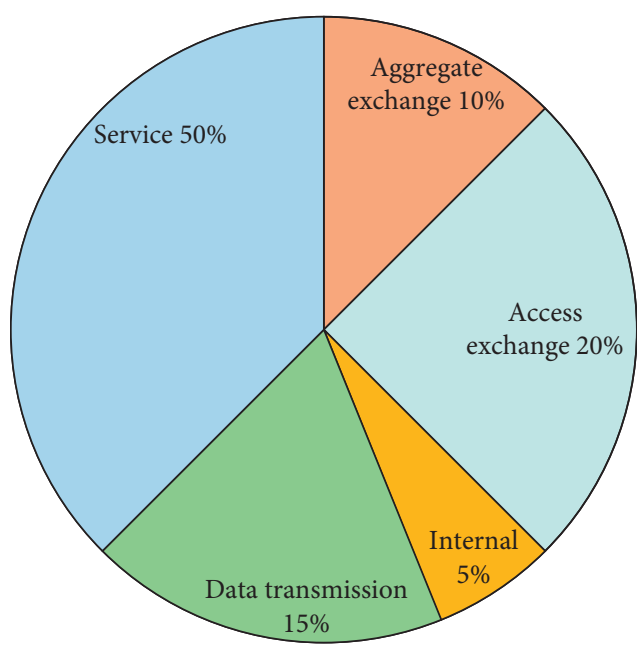

(a)

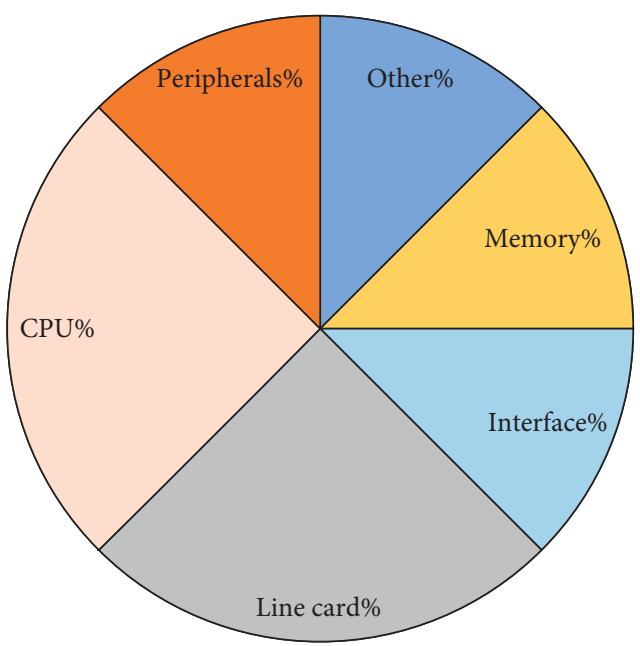

(b)

FIgURE 7: Energy consumption distribution of children's education app network. (a) Data management. (b) Terminal services.

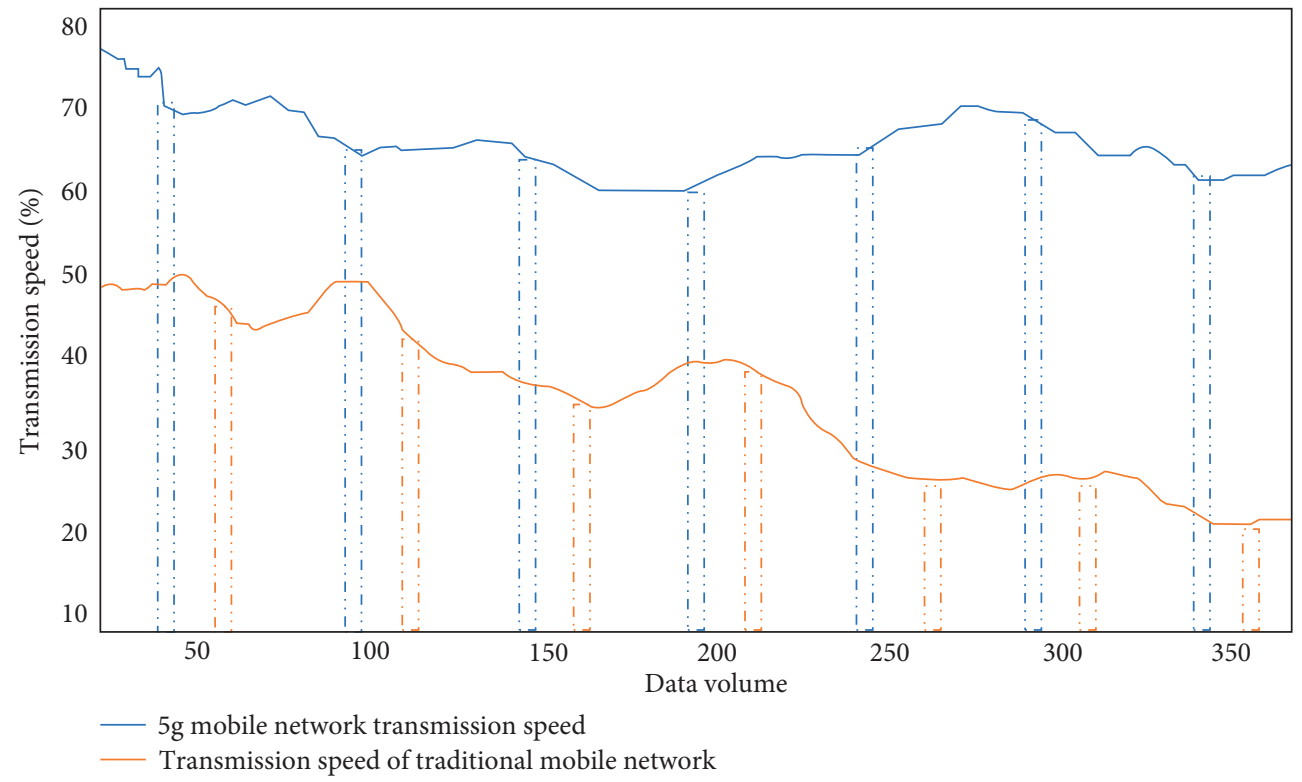

Figure 8: Comparison of transmission speed between 5G network and ordinary network.

network devices lead to large-scale interruption of mobile communication.

5G adopts the following technical countermeasures: first, strengthen the system security, track and audit the management control operation, and improve the anti attack ability. Secondly, provide end-to-end, multilevel resource security isolation measures to encrypt and backup key data. Third, strengthen the security management of open source third-party software.

With the increasing popularity of the Internet in China, the amount of data in daily contact is also increasing. In order to meet the normal use needs of educational app, we need to consider the efficiency of network transportation and select appropriate network technology for improvement. Personal information and data security need to be considered in data transmission over the mobile network, and programs with strong encryption performance should be selected in database establishment to protect private information. The $5 \mathrm{G}$ mobile network generates a large amount of data and information, and a data security mechanism is added to the network transmission process. We compare the data security of traditional mobile networks, as shown in Figure 9.

As can be seen from Figure 9, the security coefficient of traditional mobile networks gradually stabilizes with the increase of the amount of data. This security mechanism can not meet the needs in the big data environment. When using the children's education app software, we need to focus on 


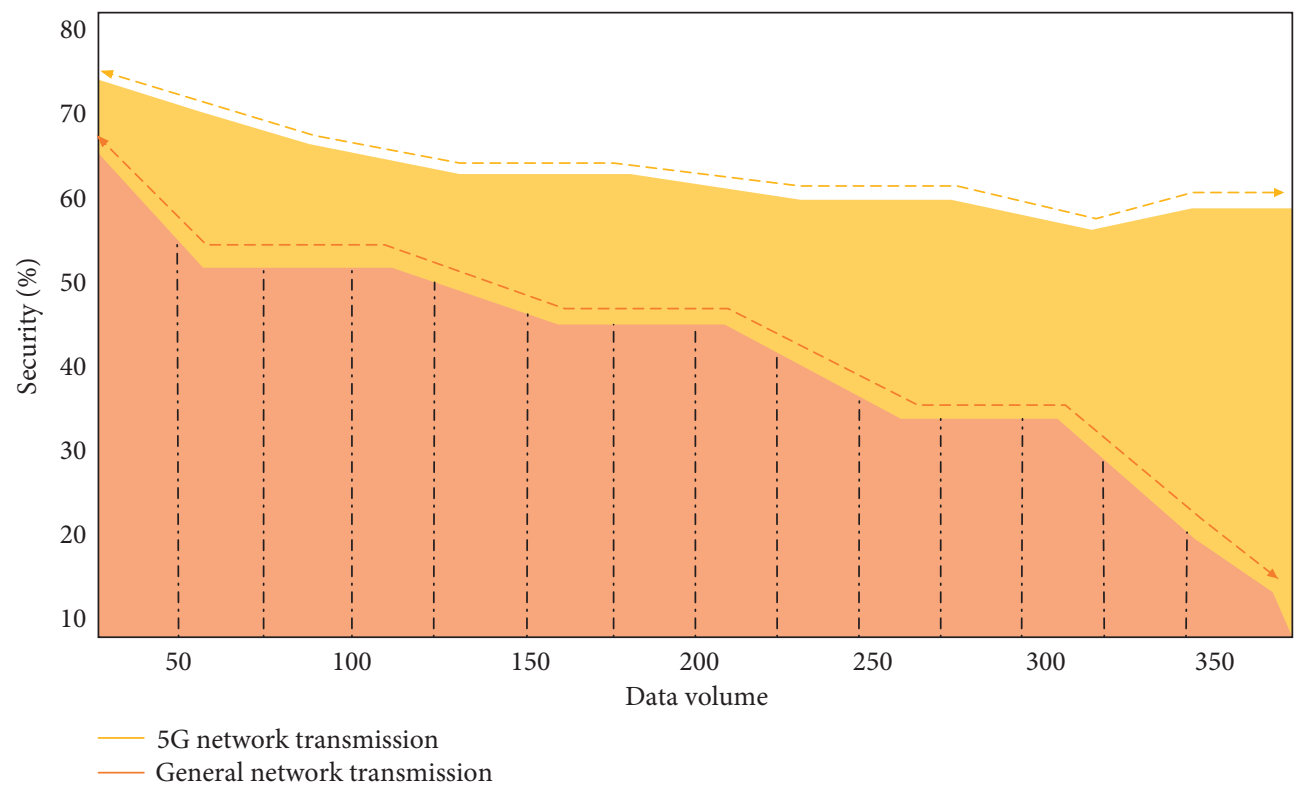

Figure 9: Comparison of data security between 5G network and traditional mobile network.

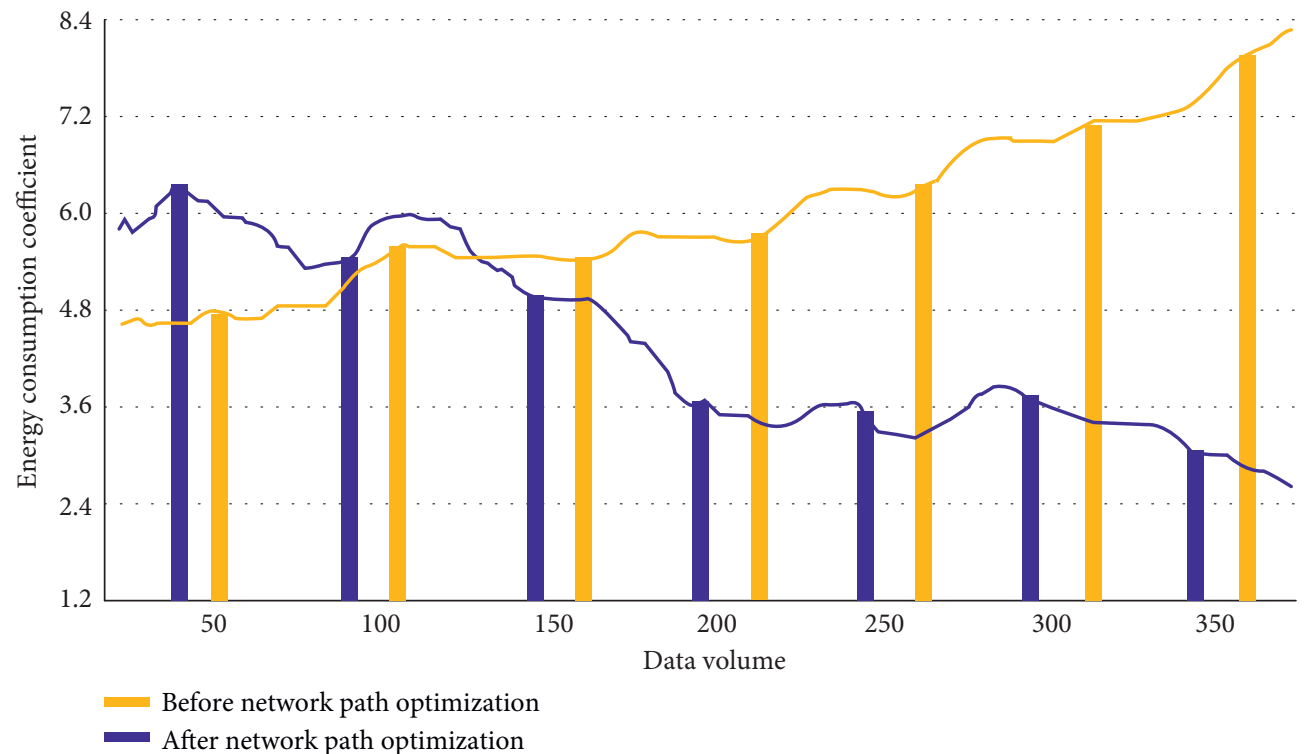

Figure 10: Changes in energy consumption of children's education app system before and after network path optimization.

the user's personal information. Therefore, the network transmission performance of $5 \mathrm{G}$ mobile computing technology has an obvious effect on the security mechanism.

\subsection{Analysis of Research Results on Path Optimization of} Children's Education App Network Based on 5G Mobile Computing Technology. We study the node location and path optimization of children's education app network under $5 \mathrm{G}$ mobile computing technology. First, we need to consider whether it can support the operation of $5 \mathrm{G}$ network in the selection of mobile terminals. In the process of user mobile node change, select the appropriate center position for signal diffusion. During this period, users within each network transmission path can receive the information released by the software. Network nodes and transmission paths form a regional network structure. When the amount of data is small, we find that the path area coverage has a negative impact. In order to study the impact of environment on path optimization, we need to monitor applications and network nodes from a variety of performance. In the subsequent research, it is found that the optimal network transmission path will change the energy consumption. The resources consumed by network transmission nodes need to be less than those consumed by data transportation. This paper selects the way of data collection in network path optimization to reduce the energy consumption in the transmission process. In the face of the data preprocessing 
behavior of transmission nodes, effective energy consumption optimization is realized while ensuring the amount of data and transmission times. Compare the energy consumption changes of children's education app system before and after network path optimization, as shown in Figure 10.

It can be seen from Figure 10 that before the network path optimization, the energy consumption of each function has exceeded the average coefficient. When we use data set to remove complex useless information, we can reduce unnecessary consumption links and improve the performance of the system. This data set structure can supplement the applications under different network coverage, improve the utilization efficiency of network frequency, and play a positive role in the network performance in $5 \mathrm{G}$ environment.

\section{Conclusion}

With the popularization of Internet and mobile communication technology, the traditional mobile network can not meet the needs of intelligent devices. With the gradual improvement of people's daily living standards, the masses pay more and more attention to the field of education. Children's education has always been the main content of attention. When modern technology is combined with children's education, many distance teaching platforms and application software emerge one after another. We study the network transmission design and network path optimization of children's education app under 5G mobile computing technology. First, the application environment and advantages of $5 \mathrm{G}$ mobile network are analyzed, and the functional requirements of children's education app are briefly described. From the perspective of students and parents, different application functions are designed, respectively, and the network transmission model is established in each function module. Using the real-time nature of data transmission to change the transmission delay rate, the hierarchical data transmission algorithm is used to obtain the optimal transmission path from the distance of network nodes. Continue to analyze the network transmission from the average efficiency, node coverage, and feedback efficiency. Finally, the security mechanism in data transmission is analyzed, and the transmission standard with protecting personal privacy as the core is selected as the support. The optimal transmission path is found by network node allocation, and the change of energy consumption in the transmission process is analyzed. The results show that in the process of children's education app network transmission under 5G mobile computing technology, the user's personal information can be guaranteed and the system feedback efficiency and operation performance can be improved. It has obvious advantages in the change of delay rate and realizes the purpose of reducing the overall energy consumption of the system.

\section{Data Availability}

The data used to support the findings of this study are available from the corresponding author upon request.

\section{Conflicts of Interest}

The authors declare that they have no conflicts of interest.

\section{References}

[1] Y. Yang, "Analysis of network transmission technology based on $5 \mathrm{G}$ ultra real time," Network security technology and application, vol. 2021, no. 11, pp. 72-73, 2021.

[2] J. Li, "Innovative design and practice of detainee mobile supervision system supported by $5 \mathrm{G}$ network," Police technology, vol. 2021, no. 6, pp. 30-33, 2021.

[3] $1 \mathrm{Li}$, "Application of $5 \mathrm{G}$ technology in civil aviation air traffic control service transmission network," China Aviation weekly, vol. 18, no. 42, pp. 60-61, 2021.

[4] B. Qin, "Research on multi robot cooperation technology based on 5G," Modern navigation, vol. 12, no. 5, pp. 363-366, 2021.

[5] X. Liu, "Research on the construction strategy of 5G communication network for operators," China new communication, vol. 23, no. 19, pp. 5-6, 2021.

[6] P. Wang, "UAV communication control system based on 5G network," Modern industrial economy and informatization, vol. 11, no. 9, pp. 147-148, 2021.

[7] X. Xu, Y. Ni, and M. Sun, "5G signal transmission train set network scheme based on network slice," Radio and television network, vol. 28, no. 9, pp. 28-30, 2021.

[8] J. Wang, "Exploration of cable TV transmission under the background of 5G era," News communication, no. 18, pp. 115-116, 2021.

[9] Z. Zhou, "Distributed UHD video processing system for 5G network," Television technology, vol. 45, no. 9, pp. 1-4, 2021.

[10] C. Lei and S. Li, "Research on application mechanism of immersive $3 \mathrm{D}$ video transmission to public security under the background of 5G network," Chinese market, vol. 22, no. 26, pp. 118-119, 2021.

[11] c Zhou, "Research on 5G mobile communication transmission network construction," Science and technology and innovation, vol. 6, no. 17, pp. 76-77, 2021.

[12] S. Q. Qian, "Technical analysis of 5G communication transmission bearer network framework," Digital communication world, vol. 2021, no. 9, pp. 133-134, 2021.

[13] J. Gao, "Analysis of optical fiber communication network transmission technology," Information and computer (theoretical Edition), vol. 33, no. 16, pp. 176-178, 2021.

[14] N. Xiao, "Discussion on ensuring data transmission reliability in 5G Communication," China new communication, vol. 23, no. 16, pp. 15-16, 2021

[15] Y. Liang, "5G technology practice and research," Metallurgical automation, vol. 45, no. S1, pp. 258-261, 2021.

[16] R. Wang, "Analysis of network information security strategy in 5G era," Software, vol. 42, no. 8, pp. 116-118, 2021.

[17] B. Wu, Y. Yang, and X. Zhang, "Design of highly reliable data transmission terminal based on $5 \mathrm{G}$ technology," Information technology and network security, vol. 40, no. 8, pp. 78-83, 2021.

[18] W. Tao, "Scheme research and field test of 5G communication network carrying CBTC system service," Urban rail transit research, vol. 24, no. 8, pp. 150-155, 2021.

[19] X. Chen, J. she, A. Wang, and H. Tang, "Research on automatic control system of railway crossing in mining area based on 5G technology," Computer knowledge and technology, vol. 17, no. 21, pp. 143-145, 2021. 
[20] J. Xi, "Research and Exploration on 5G network information security threat and protection technology," Digital technology and application, vol. 39, no. 7, pp. 178-180, 2021.

[21] S. Huang, "On the application and thinking of "learning power" app in children's education," Southeast communication, no. 8, pp. 110-111, 2021.

[22] Z. Yu, R. Liu, and L. Xiang, "Design and implementation of children's Pinyin card based on AR technology," Computer programming skills and maintenance, vol. 5, no. 7, pp. 139141, 2021.

[23] H. Q. Kun, "Research on app for preschool children's enlightenment education based on emotional design," Public standardization, no. 14, pp. 140-141, 2020.

[24] R. Zhou and Z. He, "Research on Interactive Narrative in language education app based on children's characteristics," Journal of Qiqihar University (PHILOSOPHY AND SOCIAL SCIENCES EDITION), vol. 24, no. 4, pp. 180-184, 2021.

[25] C. Duan, X. Zhao, Y. Liu, and F. Wang, "App Design of forest museum guide system for children," Design, vol. 33, no. 5, pp. 44-46, 2020. 